\title{
Mg Delta-Doping Effect on a Deep Hole Center Related to Electrical Activation of a p-Type GaN Thin Film
}

\author{
Hyo Yeol Park \\ Department of Semiconductor Applications, Ulsan College, Ulsan 680-749, Republic of Korea
}

Kyoung Nam Jeon

Division of R\&D, Kodenshi Korea Corporation, Iksan 570-210, Republic of Korea

\author{
Keunjoo Kim ${ }^{\dagger}$ \\ Department of Mechanical Engineering and Research Center of Industrial Technology, Chonbuk National University, \\ Jeonju 561-756, Republic of Korea
}

Received November 12 2009, Accepted January 72010

\begin{abstract}
The authors investigated the photoluminescence $(P L)$ and the electron paramagnetic resonance (EPR) from an magnesium (Mg)-doped GaN thin film with a delta-doped layer. The regularly doped sample shows a PL peak at $2.776 \mathrm{eV}$ for the as-grown sample, and the peak shifts to $2.904 \mathrm{eV}$ and increases in intensity for the annealed sample. The deltadoped sample also shows the same PL peak as does the regularly doped sample. However, only the annealed deltadoped layer shows a sharp EPR with a small isotropic Lande g-factor, $g_{\|}$, of 2.029. This resonance is attributed to the delta-doped layer, which forms a hole-bound $\mathrm{Mg}-\mathrm{N}$ atomic structure instead of the $\mathrm{Mg}_{\mathrm{Ga}}-\mathrm{V}_{\mathrm{N}}$ defect complex, indicating that the delta-doped sample was not optically activated to form PL centers but was instead electrically activated to form a hole-bound state.
\end{abstract}

Keywords: Delta doping process, Mg-doped GaN, Photoluminescence, Eletron paramagnetic resonance

\section{INTRODUCTION}

A great deal of research has been devoted to the p-type activation and defect nature of magnesium (Mg)-doped GaN epilayers because the p-type doping process is correlated to the low hole carrier concentration problem and issues with the mobility in p-type ohmic contact layers of optoelectronic devices [1]. It has been suggested that the Mg-H complex with an $\mathrm{Mg}$ acceptor can be compensated for by the role of hydrogen as a donor [2]. The incorporation of a hydrogen atom makes the thin film highly resistive so that there is no pathway for deriving holes in the quantum well active layer. Furthermore, due to the large approximately $260 \mathrm{meV}$ binding energy of the $\mathrm{Mg}$ acceptor, only a small fraction of all electrically active $\mathrm{Mg}$ acceptors donate a hole at room tem-

\footnotetext{
+ Author to whom corresponding should be addressed: electronic mail: kimk@chonbuk.ac.kr
}

perature [3]. This behavior of a low solid solution is typical in wide band gap semiconductors and limits their application to optoelectronic devices [4], [5].

In order to overcome this problem, Mg delta-doped GaN thin films have been intensively investigated [6]-[8]. The delta doping process is effective in the enhancement of conduction and in the reduction of dislocation density due to growth interruption so that point-density distributions of both $\mathrm{Mg}$ and Si impurities can be localized for p-type and n-type GaN layers in thin films, respectively [6], [9]. This growth interruption causes the termination of the dislocation propagation and a change in the growth polarity. The reduction of impurity self-compensation and the enhancement of free carrier concentration are also achieved by delta doping [7]. It is desirable to investigate the defect structure of the delta-doped film compared to that of the uniformly doped film [8].

In this work, we investigated the defect structure of the $\mathrm{Mg}$ impurity center in the Mg delta doped sample using photoluminescence $(\mathrm{PL})$ and electron paramagnetic resonance 


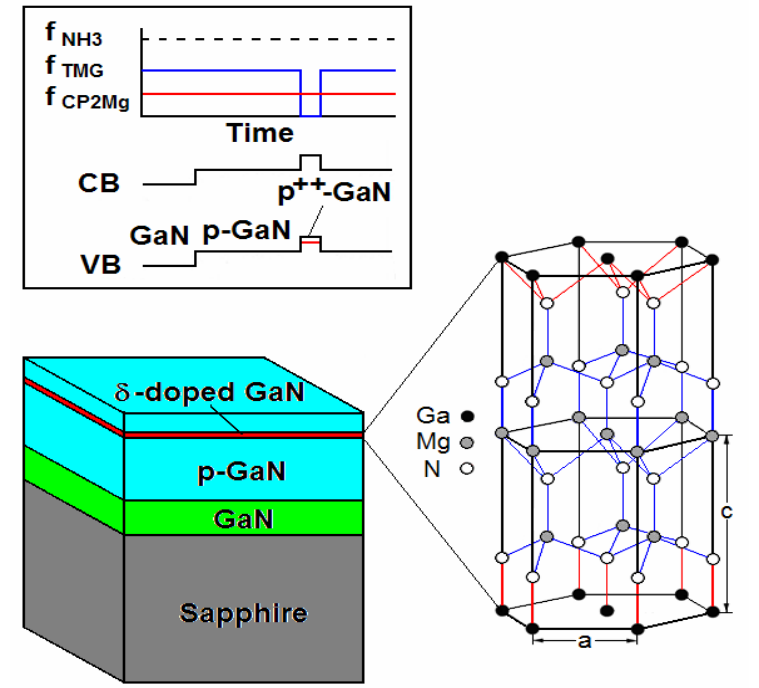

Fig. 1. Schematic diagram of heavily Mg-doped GaN epitaxial growth with the inclusion of an Mg delta-doped layer by interrupting the flow rate of the TMGa source.

(EPR) measurements. The delta-doped thin film shows a PL peak at $2.95 \mathrm{eV}$ for both the as-grown and the post-annealed samples and results in a small Lande g-factor of 2.029 for the annealed sample.

\section{EXPERIMENTS}

Figure 1 shows a schematic diagram of the heavily $\mathrm{Mg}$ doped GaN epitaxial growth layer. During the heavy Mg doping process, an inclusion of the $\mathrm{Mg}$ delta-doped layer was carried out by interrupting the flow rate of the trimethylgallium (TMGa) source. The sapphire substrate was cleaned with an acetone solution after the surface was etched by a mixed solution of $\mathrm{H}_{3} \mathrm{PO}_{4}$ and $\mathrm{H}_{2} \mathrm{SO}_{4}$. The substrate was thermally etched in a hydrogen ambient at $1,100^{\circ} \mathrm{C}$ in a metal-organic chemical vapor deposition (MOCVD) reactor for $10 \mathrm{~min}$. prior to the formation of the nucleation layer at the substrate surface. Nitridation was carried out by supplying $\mathrm{H}_{2}$ with preheated $\mathrm{NH}_{3}$ gas. As shown in Fig. 1, a 30-nm thick nucleation layer was introduced, at a low temperature of $520^{\circ} \mathrm{C}$, for geometrical bonding at the sapphire surface due to the large lattice mismatch between the sapphire substrate and the GaN film. Then, high-temperature epitaxial growths of uniformly Mg-doped GaN films with a thickness of $0.2 \mu \mathrm{m}$ at $1,100^{\circ} \mathrm{C}$ were performed by adding the $\mathrm{Mg}$ doping source of $\mathrm{Cp}_{2} \mathrm{Mg}$ into the main stream of hydrogen, ammonia, and TMGa.

In addition to this regular $\mathrm{Mg}$ doping, the $\mathrm{Mg}$ delta-doping via an instantaneous termination of the TMGa source was performed near the surface of the GaN film. We grew two samples with a uniformly Mg-doped GaN layer (uniform double layer [UDL] sample) with a thickness of $0.2 \mu \mathrm{m}$ and a uniformly Mg-doped GaN layer with the same thickness and the inclusion of an $\mathrm{Mg}$ delta-doped layer (UDL/diffuse double layer [DDL] sample) via growth interruption near the surface. These two types of Mg-doped films were thermally annealed
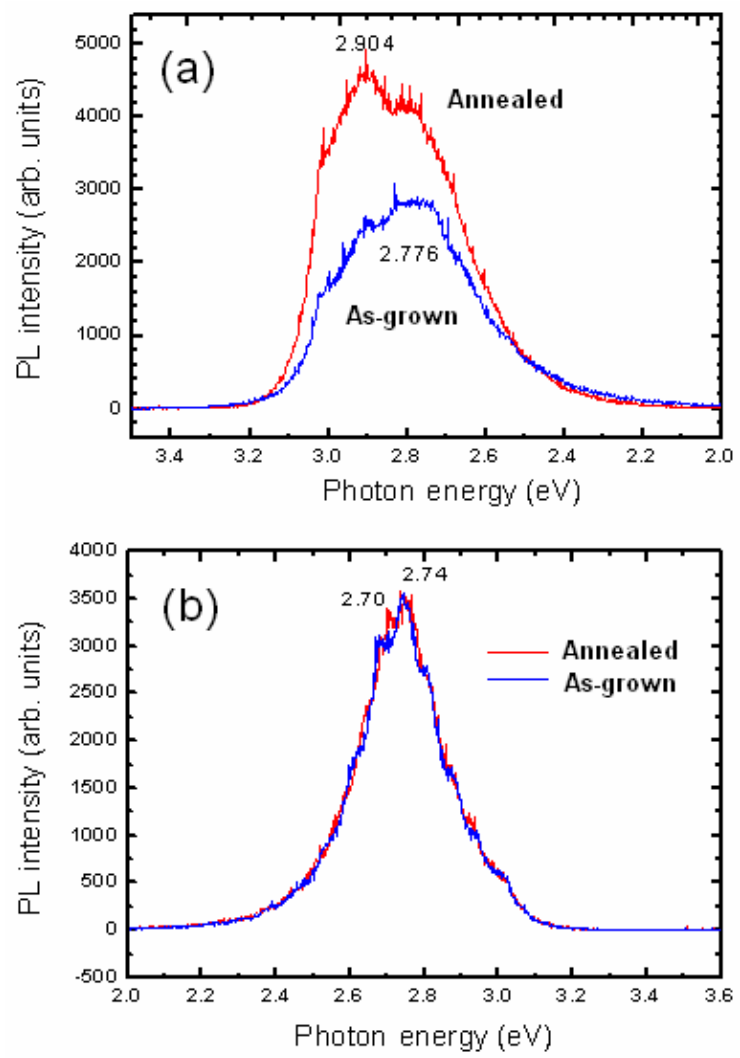

Fig. 2. The room-temperature photoluminescense $(\mathrm{PL})$ spectra from (a) the heavily Mg-doped GaN sample and (b) the heavily Mg-doped $\mathrm{GaN}$ with the inclusion of the Mg delta-doped layer. The Mg deltadoped sample shows a highly resistive film.

in a vacuum chamber at a temperature of $850^{\circ} \mathrm{C}$ for $20 \mathrm{~min}$. The Hall effect measurement for the annealed sample of the uniformly doped thin film showed a carrier concentration and hole mobility of $2.58 \times 10^{17} / \mathrm{cm}^{3}$ and $10 \mathrm{~cm}^{2} / \mathrm{V}$-sec, respectively.

The EPR measurement was performed in a Bruker 300 ESP $X$-band $(9.5 \mathrm{GHz})$ spectrometer equipped with a liquid-helium flow cryostat at a temperature of $4 \mathrm{~K}$. The background EPR signals due to the sapphire substrate were separated from the thin film signals of the acceptor lines. Room-temperature $\mathrm{PL}$ measurements were performed on each sample at $325 \mathrm{~nm}$ of a He-Cd laser for excitation.

\section{RESULTS AND DISCUSSION}

Figure 2 shows the room-temperature PL spectra from both the UDL and UDL/DDL samples. The as-grown UDL sample shown in Fig. 2(a) displays the typical blue emission peak at $2.776 \mathrm{eV}$, which is correlated with a relatively low concentration of $\mathrm{Mg}$ doping. This is attributed to a transition from the conduction band to a deep level of an $\mathrm{Mg}$ acceptor about 0.5-0.55 eV above the valence band [10]. When annealed at a temperature of $850^{\circ} \mathrm{C}$, the UDL sample showed an enhanced $\mathrm{PL}$ intensity with an extra PL peak at $2.904 \mathrm{eV}$, indicating a typical PL band for the heavily doped GaN sample [11]. For the room-temperature $\mathrm{PL}$ spectra from the UDL/DDL sample shown in Fig. 2(b), the as-grown UDL/DDL sample shows the 

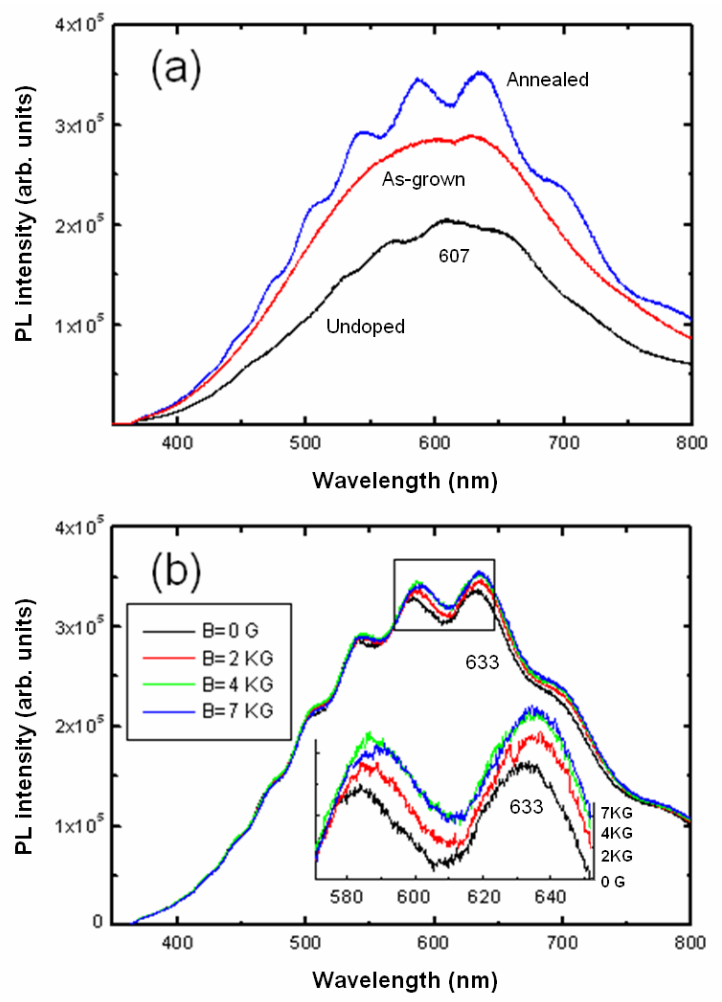

Fig. 3. The magnetic field dependence of the photoluminescense $(\mathrm{PL})$ spectra for (a) various samples of $\mathrm{GaN}$ thin films at a magnetic field of $B=4 K G$ and (b) the annealed Mg-doped sample under various magnetic fields. The PL spectra were measured at room temperature.

$\mathrm{PL}$ peak at $2.74 \mathrm{eV}$. This results from the uniformly doped layer, as was seen with the as-grown UDL sample. However, the annealed UDL/DDL sample shows the same PL peak location, but the intensity is decreased.

The annealing effect at the temperature of $850^{\circ} \mathrm{C}$ enhanced the $\mathrm{PL}$ intensity by increasing the PL centers for the regularly doped UDL sample, but it was not effective in increasing the $\mathrm{PL}$ centers from the delta-doped UDL/DDL sample. During the delta-doping process at high temperature, $1,050^{\circ} \mathrm{C}$, the atomic bonding structure of the delta-doped layer was modified and changed the growth conditions. The $\mathrm{N}$ species can be expelled from the potential barrier in the growth mechanism via introduction of the $\mathrm{Mg}$ species in the delta-doping process. This may also imply a strong reduction in the Ga vacancies, and the changed growth conditions enhanced the formation of $\mathrm{N}$ vacancies, resulting in a quench effect on the PL centers. The PL quenching is influenced by the negative electron affinity due to strong atomic bonds in the UDL/DDL sample [12].

For the heavily Mg-doped GaN sample, several defect models were suggested such as interstitial $\mathrm{Mg}$ incorporated with nitrogen vacancies $\left(\mathrm{Mg}_{1}-\mathrm{V}_{\mathrm{N}}\right)$ under the Ga-rich ambient [13] and Ga-substitutive $\mathrm{Mg}$ atoms incorporated with $\mathrm{N}$-vacancies $\left(\mathrm{Mg}_{\mathrm{Ga}}-\mathrm{V}_{\mathrm{N}}\right)$ under the $\mathrm{N}$-rich ambient [14]. As the annealing process is applied, the hydrogen does not form a bond with the $\mathrm{Mg}$ atoms but prefers the anti-bonding site of one of the $\mathrm{N}$ neighbors, resulting in the formation of $\mathrm{N}-\mathrm{H}$ bonds [14], [15]. The $\mathrm{H}$ atoms donate electrons to the $\mathrm{Mg}$ acceptor level
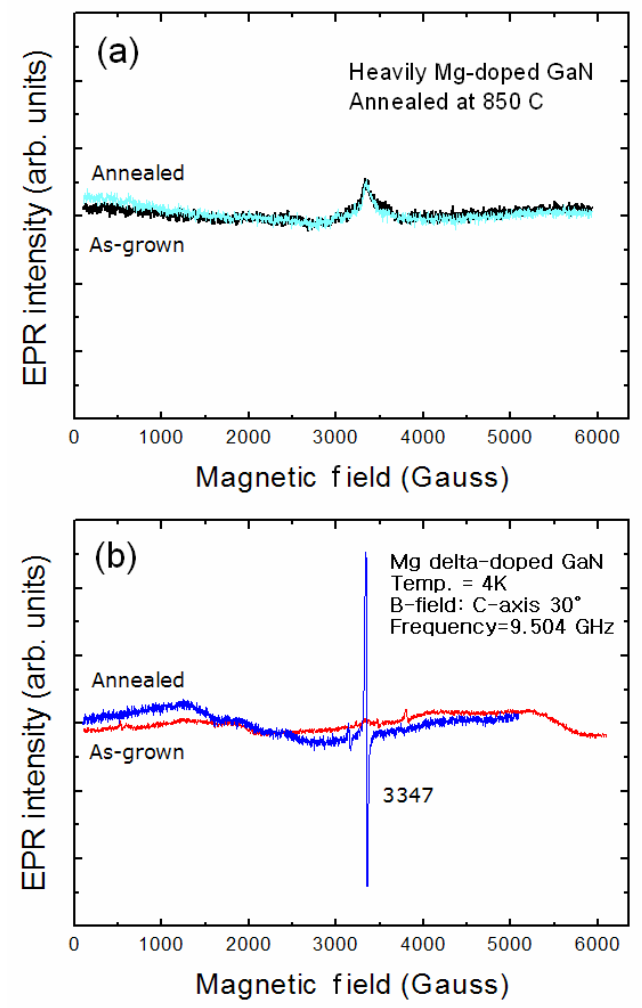

Fig. 4. The electron paramagnetic resonance (EPR) signals for both the regularly $M g$-doped UDL sample and the $M g$ delta-doped uniform/diffuse double layer (UDL/DDL) samples at low temperature, 4 $K$. The annealed delta-doped UDL/DDL sample shows EPR resonance. The as-grown Mg delta-doped GaN film does not show the EPR signal, and the heavily Mg-doped GaN films for both the as-grown and annealed UDL samples do not show EPR signals due to the low spin density at a thickness of $0.2 \mu \mathrm{m}[16]$.

and form $\mathrm{p}$-like orbitals located on the $\mathrm{N}$ atoms surrounding the Mg acceptor. The highest charge density at the bonding center includes unpaired electrons very close to $\mathrm{N}$, and the anti-bonding site of $\mathrm{N}$ forms a bond with $\mathrm{H}$. Therefore, in the delta-doped layer, the hole-bound $\mathrm{Mg}-\mathrm{N}$ atomic structure can be established. Study on the magnetic field dependency of PL centers has previously been carried out.

Figure 3 shows the magnetic field dependence of the PL spectra. Under a magnetic field of $4 \mathrm{KG}$ in Fig. 3(a), the undoped sample shows a peak at $607 \mathrm{~nm}$ that is strongly related to the dislocation defect-induced PL. The as-grown UDL/DDL sample shows an enhanced intensity, and the annealed sample shows further enhancement. The annealed sample also shows a strong oscillation at the surface, indicating Fabry-Perot interference of the GaN thin film. For the annealed UDL/DDL sample shown in Fig. 3(b), the magnetic field effect on the PL intensity is very small, but the peaks can be red-shifted as the magnetic field is increased. Without the magnetic field, the UDL/DDL sample shows a PL peak at 632.5 $\mathrm{nm}$, and as the magnetic field increases to 2,4 , and $7 \mathrm{KG}$, the peak shifted to $634.4,635.1$, and $636 \mathrm{~nm}$, respectively.

Figure 4 shows the EPR spectra for both the UDL and UDL/DDL samples. The regularly doped UDL sample in Fig. 4(a) shows no EPR spectra for either the as-grown or the annealed samples. This indicates that the as-grown UDL sam- 

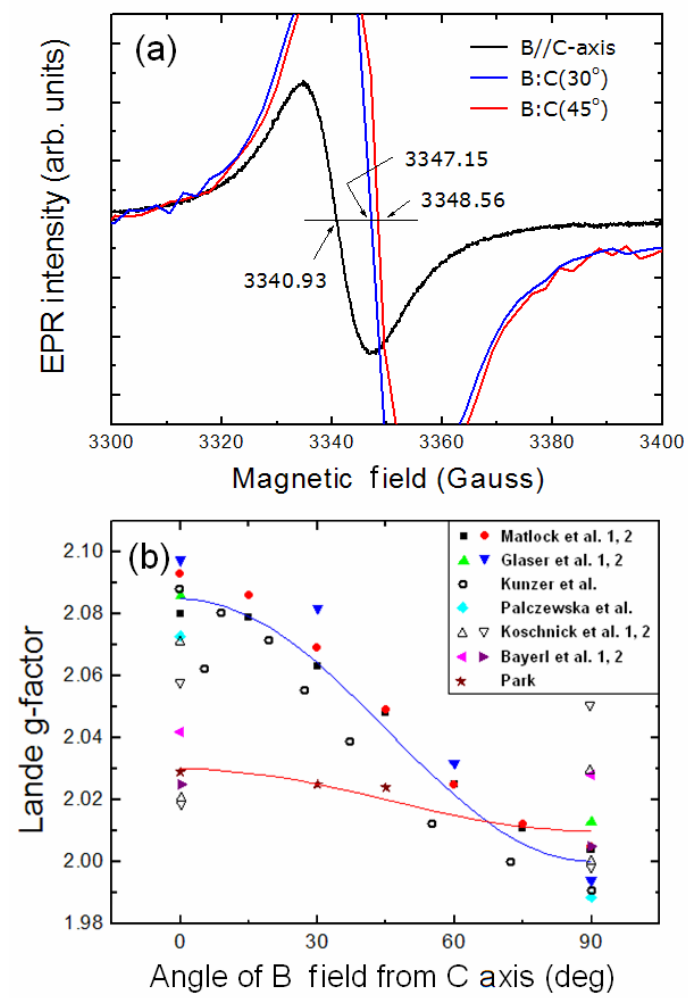

Fig. 5. The electron paramagnetic resonance (EPR) signals for various directional orientations measured from the annealed $\mathrm{Mg}$ deltadoped thin film at low temperature, $4 \mathrm{~K}$ [16], and various Lande gfactors of $\mathrm{Mg}$ dangling bonds in $\mathrm{GaN}$ as a function of the angle between the magnetic field and the $\mathrm{c}$ axis. The data were classified into two groups indicating two types of deep hole centers.

ple provides no unpaired electrons during the formation of the Mg-N-H complexes, and especially, the annealed UDL sample with the thin film thickness of $0.2 \mu \mathrm{m}$ did not provide sufficient hole spin density to show a signal in our experimental setup. However, Park reported a sharp EPR spectrum from the UDL/DDL sample [16], as shown in Fig. 4(b). The EPR spectrum appears in the annealed sample, but not in the asgrown sample. The observed EPR spectra is very sharp and narrow, indicating that the spin density is very large and similar to the defect signal from the bulk semiconductor material due to the delta doping process.

For angular variation in the EPR resonance [16], the $\mathrm{Mg}$ delta-doped sample in Fig. 5(a) shows a sharp Lorentian resonance line observed at $B=3347.1 \mathrm{G}$ at an angle of $30^{\circ}$ from the normal direction of $\mathrm{C}(0001)$ plane with a full width at half maximum (FWHM) of $17.3 \mathrm{G}$. The microwave frequency for resonance is about $9.504 \mathrm{GHz}$ for the measurement at an angle of $30^{\circ}$. The resonant position of the applied magnetic field has the following relationship: $B_{0}=h v / g(\theta) \mu_{B}$ and $g(\theta)=\left(g / / \cos ^{2} \theta+g \perp^{2} \sin ^{2} \theta\right)^{1 / 2}$, where $v$ is the resonance frequency, $\mu_{B}$ is the Bohr magneton $\left(9.2749 \times 10^{-28} \mathrm{~J} / \mathrm{G}\right), \mathrm{h}$ is the Planck constant $\left(6.6261 \times 10^{-34} \mathrm{~J} \cdot \mathrm{s}\right), \mathrm{g}(\theta)$ is the Lande g-factor corresponding to the angle $\theta$ of the magnetic field $B_{0}$ from the $c$-axis. The derived g-value is $g\left(30^{\circ}\right)=2.026(1)$. Fig. $5(a)$ shows the very slight anisotropic behavior of the EPR signals. For the microwave resonance frequencies of 9.502, 9.504, and $9.491 \mathrm{CHz}$, the g-values at $0^{\circ}, 30^{\circ}$ and $45^{\circ}$ are obtained as
2.029(5), 2.026(1) and 2.022(5) with FWHMs of about 13.2, 17.3, and $18.1 \mathrm{G}$, respectively. The derivative g-value at the right angle is about $g \perp=2.014(5)$. This g-value is still larger than that of a free electron, $g_{e}=2.0023$, as expected for most free acceptors. The intense signal at an angle of around 30$45^{\circ}$ is consistent with the axis of the Mg-N bond line [17].

The uniformly doped thick films have been known to yield very broad EPR signals [18]-[20]. As shown in Fig. 5(b), these films showed relatively large g-values around $g_{\|}=2.09-2.10$. Because of the low hole carrier concentration in the Mgdoped GaN thin film, a very weak EPR signal from the unpaired dangling bonds has been observed for $g_{\|}$values of 2.10 [18]. Optically detected magnetic resonance has revealed several Mg-related recombination centers with reported gvalues of $g_{\|}=2.08$ and $g \perp=2.00$ for uniformly Mg-doped GaN sam ples. The uniformly doped samples with a PL band peaked around $2.79 \mathrm{eV}$ and also revealed an EPR signal with large values of $g_{\|}=2.073-2.112$ with an FWHM of about $140 \mathrm{G}$ [19]-[21]. The thick epitaxial films have the resonance behavior with weak intensity with a broad FWHM due to the limited solid solution of Mg dopants.

According to Koschnick et al. [22], an Mg-doped sample shows a PL-EPR resonance with $g_{\|}=2.07, g_{\perp}=2.03$ having a broad PL at $2.74 \mathrm{eV}$, and the highly resistive sample showed a $\mathrm{PL}-E P R$ resonance with $\mathrm{g}_{\|}=2.057, \mathrm{~g} \perp=2.045$ having broad $\mathrm{PL}$ at $3.12 \mathrm{eV}$. The PL-EPR resonance of $g_{\|}=2.07, \mathrm{~g} \perp=2.03$ in this study was similar to those observed by Glaser et al. [19] and Kunzer et al. [20] and that assigned to a deep Mg-related complex. There is another report on relatively small values, $\mathrm{g}_{\|}=2.025-2.042$ and $\mathrm{g} \perp=2.005-2.028$ with a FWHM of about $150 \mathrm{G}$, in electrically detected magnetic resonance [23]. This indicates that the Mg-doped samples can be classified into two groups based on their large or small g-values.

A large $g$ factor represents the defect properties of a broad EPR spectrum with large PL centers for the $\left(\mathrm{Mg}_{\mathrm{Ga}}-\mathrm{V}_{\mathrm{N}}\right)$ defect complex. The EPR signal was quenched at the high annealing temperature of $850^{\circ} \mathrm{C}$. The delta-doped g-values were assigned to the small value group, although there was a large difference in the FWHMs. The very small FWHMs of the EPR spectra signal from the delta-doped layer were similar to the signal in the bulk material, rather than that of the epitaxial film. The EPR signal was very strong even at an annealing temperature of $850^{\circ} \mathrm{C}$. However, the defect center did not reserve $\mathrm{PL}$ centers.

This delta-doping effect can be speculated from the atomic structure of the defect site in the crystal, as shown in Fig. 6. For the heavily doped UDL sample, the as-grown sample formed a defect site with the cooperation of hydrogen bonds as shown in Fig. 6(a). The annealed UDL sample formed the $\mathrm{Mg}_{\mathrm{Ga}}-\mathrm{V}_{\mathrm{N}}$ complex with the $\mathrm{NH}_{3}$ molecular species over a range of annealing temperatures from 600 to $850^{\circ} \mathrm{C}$ as shown in Fig. $6(\mathrm{~b})$. For temperatures higher than $850^{\circ} \mathrm{C}$, the $\mathrm{NH}_{3}$ molecular species can diffuse from the substance. This defect site forms the $\mathrm{PL}$ center with both the $\mathrm{Mg}_{\mathrm{Ga}}$-acceptor and the $V_{N}$-donor [24]. However, the hole density of the defect site is very broad and shows a large EPR signal and a broad PL spectra, indicating the potential fluctuation.

For the delta-doped UDL/DDL sample, the as-grown sample formed a defect site with a nitrogen vacancy, which was saturated by a hydrogen bond and interstitial molecules as 

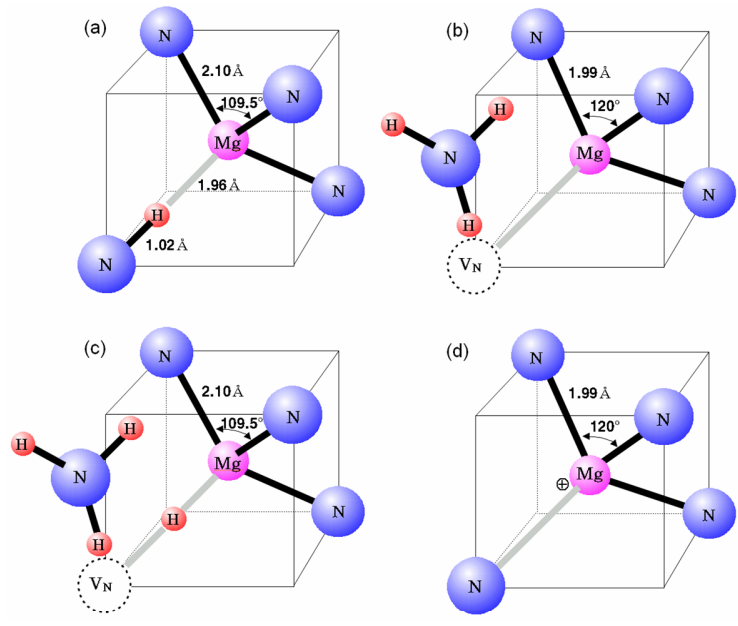

Fig. 6. Atomic force microscopy image of the nanopatterned surface of the n-GaN thin film. The nanopattern was processed by ebeam nanolithography with a photonic crystal lattice of $230 \mathrm{~nm}$. The nanoholes have a diameter of $112 \mathrm{~nm}$ and a depth of $30 \mathrm{~nm}$.

shown in Fig. 6(c). The hydrogen saturated defect site showed no EPR signal, but the PL centers were still active. For the annealing process at a temperature of $850^{\circ} \mathrm{C}$, the defect site formed nitrogen antibonding with the dissociation of hydrogen gas as shown in Fig. 6(d). This defect site showed a hole center from the EPR signal, and the no donor site had no PL centers. For a monolayer of $\mathrm{Mg}$ atoms in the GaN thin film, the atomic density was $6.81 \times 10^{15} / \mathrm{cm}^{2}$ and a sufficient electron spin density was provided by the $\mathrm{Mg}$ dangling bonds, which yielded a relatively sharp EPR peak. The Mg delta-doping process cannot enhance the PL centers but is useful for the formation of a hole channel in the Ohmic metal contact area.

\section{CONCLUSIONS}

In summary, the delta-doping effect on Mg-doped GaN thin film was investigated in terms of the PL and EPR characteristics. The as-grown UDL/DDL sample showed PL bands peaked at $2.74 \mathrm{eV}$, identical to the annealed sample. The as-grown sample also showed either the same or lower PL intensity as that of the annealed sample after the post-thermal annealing process. Therefore, the delta-doping was not effective in enhancing the PL centers. Furthermore, the EPR signal from the annealed UDL/DDL sample resulted in a relatively small $g$ factor of $g_{\|}=2.029$ compared to the literature values of about $\mathrm{g}_{\|}=2.09$ from relatively thick and uniformly Mg-doped GaN films. This implied that the defect mechanism of the delta-doped layer was totally different from that of the regularly doped layer. This difference may be due to the deep hole center of the Mg-N atomic structure rather than from a defect structure of the $\mathrm{Mg}_{\mathrm{Ga}}-\mathrm{V}_{\mathrm{N}}$ complex. The delta-doping process will be useful for hole channel transport on p-type GaN surfaces attempting to contact ohmic metal.

\section{REFERENCES}

[1] H. Amano, M. Kito, K. Hiramatsu, and I. Akasaki, Jpn. J. Appl. Phys. 28, L2112 (1989) [DOI: 10.1143/JJAP.28.L2112].

[2] S. Nakamura, N. Iwasa, M. Senoh, and T. Mukai, Jpn. J. Appl. Phys. 31, 1258 (1992) [DOI: 10.1143/JJAP.31.1258].

[3] K. Kim and S. J. Chung, Appl. Phys. Lett. 80, 1767 (2002) [DOI: 10.1063/1.1456547].

[4] L. Eckey, U. Von Gfug, J. Holst, A. Hoffmann, B. Schineller, K. Heime, M. Heuken, O. Schon, and R. Beccard, J. Cryst. Growth 189-190, 523 (1998) [DOI: 10.1016/s0022-0248(98)00344-3].

[5] K. Kim and S. J. Chung, Trans. Electr. Electron. Mater. 2, 24 (2001).

[6] M. L. Nakarmi, K. H. Kim, J. Li, J. Y. Lin, and H. X. Jiang, Appl. Phys. Lett. 82, 3041 (2003) [DOI: 10.1063/1.1559444].

[7] Z. Liliental-Weber, M. Benamara, W. Swider, J. Washburn, I. Grzegory, S. Porowski, D. J. H. Lambert, C. J. Eiting, and R. D. Dupuis, Appl. Phys. Lett. 75, 4159 (1999) [DOI: 10.1063/1.125568].

[8] C. Simbrunner, M. Wegscheider, M. Quast, T. Li, A. NavarroQuezada, H. Sitter, A. Bonanni, and R. Jakiela, Appl. Phys. Lett. 90, 142108 (2007) [DOI: 10.1063/1.2719171].

[9] K. Kim, Trans. Electr. Electron. Mater. 5, 173 (2004).

[10] J. M. Myoung, K. H. Shim, C. Kim, O. Gluschenkov, K. Kim, S. Kim, D. A. Turnbull, and S. G. Bishop, Appl. Phys. Lett. 69, 2722 (1996) [DOI: 10.1063/1.11769o].

[11] K. Kim and J. G. Harrison, J. Vac. Sci. Technol. A 21, 134 (2003) [DOI: 10.1116/1.1524148].

[12] C. I. Wu and A. Kahn, J. Appl. Phys. 86, 3209 (1999) [DOI: 10.1063/1.371191].

[13] S. G. Lee and K. J. Chang, Semicond. Sci. Technol. 14, 138 (1999) [DOI: 10.1088/0268-1242/14/2/006].

[14] J. Neugebauer and C. G. Van de Walle, Phys. Rev. Lett. 75, 4452 (1995) [DOI: 10.1103/PhysRevLett.75.4452].

[15] W. Gotz, N. M. Johnson, D. P. Bour, M. D. McCluskey, and E. E. Haller, Appl. Phys. Lett. 69, 3725 (1996) [DOI: 10.1063/ 1.117202].

[16] H. Y. Park, J. Korean Soc. Semicond. Disp. Equip. Technol.(in Korean) 4, 1 (2005).

[17] S. M. Myers, A. F. Wright, G. A. Petersen, C. H. Seager, W. R. Wampler, M. H. Crawford, and J. Han, J. Appl. Phys. 88, 4676 (2000) [DOI: 10.1063/1.1309123].

[18] D. Matlock, M. Zvanut, H. Wang, J. R. Dimaio, R. F. Davis, J. E. van Nostrand, R. Henry, D. Koleske, and A. Wickenden, J. Electron. Mater. 34, 34 (2005) [DOI: 10.1007/s11664-005-0177-3].

[19] E. R. Glaser, W. E. Carlos, G. C. B. Braga, J. A. Freitas, W. J. Moore, B. V. Shanabrook, R. L. Henry, A. E. Wickenden, D. D. Koleske, H. Obloh, P. Kozodoy, S. P. DenBaars, and U. K. Mishra, Phys. Rev. B 65, 085312 (2002) [DOI: 10.1103/ PhysRevB.65.085312].

[20] M. Kunzer, U. Kaufmann, K. Maier, J. Schneider, N. Herres, I. Akasaki, and H. Amano, Mater. Sci. Forum 143-147, 87 (1994) [DOI: 10.4028/www.scientific.net/MSF.143-147.87].

[21] M. Palczewska, B. Suchanek, R. Dwilinski, K. Pakula, A. Wagner, and M. Kaminska, MRS Internet J. Nitride Semicond. Res. 3, 45 (1998) http://nsr.mij.mrs.org/3/45/complete.html.

[22] F. K. Koschnick, K. Michael, J. M. Spaeth, B. Beaumont, P. Gibart, J. Off, A. Sohmer, and F. Scholz, J. Cryst. Growth 189-190, 561 (1998) [DOI: 10.1016/s0022-0248(98)00198-5].

[23] M. W. Bayerl, M. S. Brandt, H. Angerer, O. Ambacher, and M. Stutzmann, Phys. Status Solidi B 210, 389 (1998) [DOI: 10.1002/(SICI)1521-3951(199812)210:2<389::AID$\mathrm{PSSB} 389>3.0 . \mathrm{CO} ; 2-\mathrm{Y}]$.

[24] M. A. Reshchikov and H. Morkoc, J. Appl. Phys. 97, 061301 (2005) [DOI: 10.1063/1.1868059]. 